Pacific

Journal of

Mathematics

SUPPORT SETS OF PAIRS OF MODULES

DAvid A. JoRgensen 


\title{
SUPPORT SETS OF PAIRS OF MODULES
}

\author{
DAVID A. Jorgensen
}

Let $R$ be the quotient of a local domain $(Q, \mathfrak{n})$ by a proper ideal minimally generated by $f_{1}, \ldots, f_{c}$. Assume $Q / \mathfrak{n}$ is algebraically closed, and let $M$ and $N$ be finitely generated $R$ modules. We show there is an algebraic set in $c$-dimensional affine space, called the support set of the pair $(M, N)$, which describes those hypersurfaces $h \in\left(f_{1}, \ldots, f_{c}\right)-\mathfrak{n}\left(f_{1}, \ldots, f_{c}\right)$ over which there are infinitely many nonzero $\operatorname{Ext}_{Q /(h)}^{i}(M, N)$. This generalizes to arbitrary quotients of regular local rings the notion of support variety for modules over complete intersections.

\section{Introduction.}

Let $Q$ be a local ring with maximal ideal $\mathfrak{n}$ and residue field $k$. Since we will be talking about algebraic sets in affine space over $k$, we assume that $k$ is algebraically closed. ${ }^{1}$ Suppose $\boldsymbol{f}:=f_{1}, \ldots, f_{c}$ are elements of $Q$ which minimally generate their ideal $(\boldsymbol{f})$. Throughout this note we assume $(\boldsymbol{f})$ is a proper ideal and write $R$ for the quotient ring $Q /(\boldsymbol{f})$. We let $M$ and $N$ denote finitely generated $R$-modules.

When $\boldsymbol{f}$ is a $Q$-regular sequence it is known [5] that $\operatorname{Ext}_{R}^{*}(M, N)$ possesses a structure of graded module over the polynomial ring $R\left[x_{1}, \ldots, x_{c}\right]$, and this graded module is finitely generated over $R\left[x_{1}, \ldots, x_{c}\right]$ precisely when $\operatorname{Ext}_{Q}^{i}(M, N)=0$ for $i \gg 0$. Set $\mathcal{E}:=\operatorname{Ext}_{R}^{*}(M, N) \otimes_{R} k$ and $\mathcal{R}:=$ $R\left[x_{1}, \ldots, x_{c}\right] \otimes_{R} k$. In [2] Avramov and Buchweitz define the support variety $\mathrm{V}(Q, \boldsymbol{f} ; M, N)$ of a pair of modules $(M, N)$ as the zero set in $k^{c}$ of the annihilator of $\mathcal{E}$ in $\mathcal{R}$, that is

$$
\begin{array}{r}
\mathrm{V}(Q, \boldsymbol{f} ; M, N):=\left\{\left(\alpha_{1}, \ldots, \alpha_{c}\right) \in k^{c} \mid \phi\left(\alpha_{1}, \ldots, \alpha_{c}\right)=0\right. \\
\left.\quad \text { for all } \phi \in \mathrm{Ann}_{\mathcal{R}} \mathcal{E}\right\} \cup\{0\} .
\end{array}
$$

(The predecessor of support variety, called cohomological variety, is defined by Avramov in $[\mathbf{1}]$.) We write $\mathrm{V}(Q, \boldsymbol{f} ; M)$ for $\mathrm{V}(Q, \boldsymbol{f} ; M, k)$. Note that since $\mathcal{E}$ is graded, its annihilator is a homogeneous ideal, and so $\mathrm{V}(Q, \boldsymbol{f} ; M, N)$ is a cone.

\footnotetext{
${ }^{1}$ This assumption is not really necessary, but we make it in favor of a simpler exposition. See the remark at the end of this section.
} 
Support varieties offer a very useful description of the homological interrelations between resolutions of the associated modules. (See, for example, Theorem AB below.) It seems worthwhile to investigate possible generalizations of support varieties to pairs of modules over quotient rings not defined modulo a regular sequence.

In Theorem 2.5 of [2], Avramov and Buchweitz show that when $\operatorname{Ext}_{Q}^{i}(M$, $N)=0$ for $i \gg 0$, the nonzero points of $\mathrm{V}(Q, \boldsymbol{f} ; M, N)$ correspond to those hypersurfaces $h \in(\boldsymbol{f})-\mathfrak{n}(\boldsymbol{f})$ such that there are infinitely many nonzero $\operatorname{Ext}_{Q /(h)}^{i}(M, N)$. Regarding this as the definition, one may naively extend the notion of support variety to arbitrary quotient rings as follows.

Defintion 1.1. Let $R:=Q /(\boldsymbol{f})$ be an arbitrary quotient of $Q$. Define the cohomological support set $\mathrm{V}(Q, \boldsymbol{f} ; M, N)$ of the pair $(M, N)$ as

$$
\left\{\boldsymbol{\alpha} \in k^{c} \mid \text { there exists } \boldsymbol{a} \in Q^{c} \text { with } \operatorname{Ext}_{Q_{\boldsymbol{a}}}^{i}(M, N) \neq 0\right.
$$

$$
\text { for infinitely many } i\} \cup\{0\} \text {, }
$$

where $\boldsymbol{a}:=\left(a_{1}, \ldots, a_{c}\right) \in Q^{c}$ is a preimage of $\boldsymbol{\alpha}:=\left(\alpha_{1}, \ldots, \alpha_{c}\right) \in k^{c}$ and $Q_{\boldsymbol{a}}$ is the hypersurface $Q /\left(a_{1} f_{1}+\cdots+a_{c} f_{c}\right)$.

There are two important questions regarding this definition:

(1) Are the nonzero elements $\boldsymbol{\alpha} \in \mathrm{V}(Q, \boldsymbol{f} ; M, N)$ determined independently of the choice of preimage $\boldsymbol{a} \in Q^{c}$ ?

(2) Is $\mathrm{V}(Q, \boldsymbol{f} ; M, N)$ closed in $k^{c}$ ?

Theorem 2.5 of $[\mathbf{2}]$ shows that both answers are "yes" in the case where $\boldsymbol{f}$ is a $Q$-regular sequence and $\operatorname{Ext}_{Q}^{i}(M, N)=0$ for $i \gg 0$. The main point of this note (Theorem 2.1) is that both answers are also "yes" when $Q$ is a domain and $(\boldsymbol{f})$ is an arbitrary (proper) ideal of $Q$. Since, in general, $\operatorname{Ext}_{R}^{*}(M, N) \otimes_{R} k$ may not have a well-defined structure of graded module over the polynomial ring $k\left[x_{1}, \ldots, x_{c}\right]$, our proof is necessarily totally different from that of $[\mathbf{2}]$.

Another concern at this point is whether there exist nontrivial examples of cohomological support sets when $(\boldsymbol{f}) \subseteq \mathfrak{n}^{2}$, that is, examples where $\mathrm{V}(Q, \boldsymbol{f} ; M, N) \neq k^{c}$. Such nontrivial examples are well-known in the case where $\boldsymbol{f}$ is a $Q$-regular sequence. (See, for example, 6.3, 6.5 and 7.6 of [1].) Part of Sections 3 and 4 are devoted to exhibiting nontrivial examples with $f \in \mathfrak{n}^{2}$ not a regular sequence on $Q$.

When $Q$ is a regular local ring and $\boldsymbol{f}$ a $Q$-regular sequence contained in $\mathfrak{n}^{2}$ then $R$ is called a complete intersection of codimension $c$. For complete intersections the support set $\mathrm{V}(Q, \boldsymbol{f} ; M, N)$ renders precise information about the homological behavior over $R$ of the pair $(M, N)$. A good illustration of this is the following remarkable result of Avramov and Buchweitz.

Theorem AB ([2]). Let $M$ and $N$ be finitely generated $R$-modules over the complete intersection $R$. Then the following are equivalent: 
(1) $\operatorname{Ext}_{R}^{n}(M, N)=0$ for $n \gg 0$.

(2) $\operatorname{Ext}_{R}^{n}(N, M)=0$ for $n \gg 0$.

(3) $\operatorname{Tor}_{n}^{R}(M, N)=0$ for $n \gg 0$.

(4) $\mathrm{V}(Q, \boldsymbol{f} ; M) \cap \mathrm{V}(Q, \boldsymbol{f} ; N)=\{0\}$.

(The implication $(3) \Longrightarrow(4)$ was first proven in [7].)

Of course, it is impossible to extend even the equivalence (1) $\Longleftrightarrow(3)$ to non-Gorenstein rings, and so a homological counterpart to $\mathrm{V}(Q, \boldsymbol{f} ; M, N)$ is not superfluous:

Defintion 1.2. Let $R:=Q /(\boldsymbol{f})$ be an arbitrary quotient of $Q$. Define the homological support set $\mathrm{U}(Q, \boldsymbol{f} ; M, N)$ of the pair $(M, N)$ as

$$
\begin{aligned}
\left\{\boldsymbol{\alpha} \in k^{c} \mid \text { there exists } \boldsymbol{a} \in Q^{c} \text { with } \operatorname{Tor}_{i}^{Q_{a}}(M, N) \neq 0\right. \\
\\
\text { for infinitely many } i\} \cup\{0\},
\end{aligned}
$$

where $\boldsymbol{a}:=\left(a_{1}, \ldots, a_{c}\right) \in Q^{c}$ is a preimage of $\boldsymbol{\alpha}:=\left(\alpha_{1}, \ldots, \alpha_{c}\right) \in k^{c}$ and $Q_{\boldsymbol{a}}$ is the hypersurface $Q /\left(a_{1} f_{1}+\cdots+a_{c} f_{c}\right)$.

Questions (1) and (2), for homological support sets, will also be answered in the affirmative provided $Q$ is a domain (by Theorem 2.2) in the next section.

In Section 3 we give properties of the support sets and discuss which implications from Theorem AB can possibly be extended to more general quotients of regular local rings using our generalization of $\mathrm{V}(Q, \boldsymbol{f} ; M)$.

Section 4 consists of Macaulay 2 code which uses Theorem 2.2 (or Theorem 2.1) to compute the homological (or cohomological) support set of a pair of modules. We also include several examples.

Remark. The assumption made at the beginnning of this note, that the residue field $k$ of $Q$ is algebraically closed, is not really restrictive. For there is always a faithfully flat extension $Q \subseteq \widetilde{Q}$ of local rings such that $\mathfrak{n} \widetilde{Q}$ is the maximal ideal of $\widetilde{Q}$ and the residue field $\widetilde{k}$ of $\widetilde{Q}$ is an algebraic closure of $k$. (See App., Théorème 1, Corollaire of [3].) Hence the general definition of support set can be given by $\mathrm{V}(Q, \boldsymbol{f} ; M, N):=\mathrm{V}(\widetilde{Q}, \boldsymbol{f} ; \widetilde{M}, \widetilde{N})$, where $\widetilde{M}:=M \otimes_{Q} \widetilde{Q}$ and $\widetilde{N}:=N \otimes_{Q} \widetilde{Q}$. This definition is compatible with the definition given in [2] of support variety for rings $Q$ modulo a regular sequence with non-algebraically closed residue field. (See Lemma 2.2 of [2].)

\section{Support sets.}

The main results of this section are Theorems 2.1 and 2.2, which show that the support sets $\mathrm{V}(Q, \boldsymbol{f}, M, N)$ and $\mathrm{U}(Q, \boldsymbol{f}, M, N)$ are closed in $k^{c}$ and that their nonzero elements $\boldsymbol{\alpha}$ are determined independently of the choice of preimage $\boldsymbol{a}$, provided $Q$ is a domain.

Before stating the theorems we set some notation. 
Let $S$ denote the generic hypersurface $Q\left[X_{1}, \ldots, X_{c}\right] /\left(X_{1} f_{1}+\cdots+X_{c} f_{c}\right)$, where $\boldsymbol{X}:=X_{1}, \ldots, X_{c}$ are indeterminates. By abuse of notation we will write $X_{i}$ for the image of $X_{i}$ in $S$. For $a_{1}, \ldots, a_{c} \in Q$ we have $S /\left(X_{1}-\right.$ $\left.a_{1}, \ldots, X_{c}-a_{c}\right) \simeq Q_{\boldsymbol{a}}$ as rings, and $M[\boldsymbol{X}] /\left(X_{1}-a_{1}, \ldots, X_{c}-a_{c}\right) M[\boldsymbol{X}] \simeq M$ as $S$-modules. Set

$$
I_{i}:=\operatorname{Ann}_{S / \mathfrak{n} S}\left(\operatorname{Ext}_{S}^{i}(M[\boldsymbol{X}], N[\boldsymbol{X}]) \otimes_{S} S / \mathfrak{n} S\right),
$$

and

$$
J_{i}:=\operatorname{Ann}_{S / \mathfrak{n} S}\left(\operatorname{Tor}_{i}^{S}(M[\boldsymbol{X}], N[\boldsymbol{X}]) \otimes_{S} S / \mathfrak{n} S\right) .
$$

Note that $S / \mathfrak{n} S$ is isomorphic to the polynomial ring $k\left[x_{1}, \ldots, x_{c}\right]$ and that $I_{i}$ and $J_{i}$ are homogeneous for all $i$. For an ideal $I \subseteq k\left[x_{1}, \ldots, x_{c}\right]$ we let $\mathrm{Z}(I)$ denote the algebraic set in $k^{c}$ defined by $I$.

Theorem 2.1. Suppose $R:=Q /(\boldsymbol{f})$ is a quotient of a local domain $(Q, \mathfrak{n}, k)$.

(1) If $\operatorname{Ext}_{Q}^{i}(M, N) \neq 0$ for infinitely many $i$, then

$$
\mathrm{V}(Q, \boldsymbol{f} ; M, N)=k^{c} .
$$

(2) If $\operatorname{Ext}_{Q}^{i}(M, N)=0$ for all $i \geq v \in \mathbb{N}$, then for any integer $n$ satisfying $n \geq v-1$

$$
\mathrm{V}(Q, \boldsymbol{f} ; M, N)=\mathrm{Z}\left(I_{n} \cap I_{n+1}\right) \cup\{0\} .
$$

Moreover, if $\boldsymbol{a}:=\left(a_{1}, \ldots, a_{c}\right)$ and $\boldsymbol{b}:=\left(b_{1}, \ldots, b_{c}\right)$ in $Q^{c}$ are both preimages of $\boldsymbol{\alpha} \neq 0$ in $k^{c}$, then $\operatorname{Ext}_{Q_{\boldsymbol{a}}}^{i}(M, N) \neq 0$ for infinitely many $i$ if and only if $\operatorname{Ext}_{Q_{b}}^{i}(M, N) \neq 0$ for infinitely many $i$.

The analogous homological version of 2.1 is given by:

Theorem 2.2. Suppose $R:=Q /(\boldsymbol{f})$ is a quotient of a local domain $(Q, \mathfrak{n}, k)$.

(1) If $\operatorname{Tor}_{i}^{Q}(M, N) \neq 0$ for infinitely many $i$, then

$$
\mathrm{U}(Q, \boldsymbol{f} ; M, N)=k^{c} .
$$

(2) If $\operatorname{Tor}_{i}^{Q}(M, N)=0$ for all $i \geq v \in \mathbb{N}$, then for any integer $n$ satisfying $n \geq v-1$,

$$
\mathrm{U}(Q, \boldsymbol{f} ; M, N)=\mathrm{Z}\left(J_{n} \cap J_{n+1}\right) \cup\{0\} .
$$

Moreover, if $\boldsymbol{a}:=\left(a_{1}, \ldots, a_{c}\right)$ and $\boldsymbol{b}:=\left(b_{1}, \ldots, b_{c}\right)$ in $Q^{c}$ are both preimages of $\boldsymbol{\alpha} \neq 0$ in $k^{c}$, then $\operatorname{Tor}_{i}^{Q \boldsymbol{Q}}(M, N) \neq 0$ for infinitely many $i$ if and only if $\operatorname{Tor}_{i}^{Q_{b}}(M, N) \neq 0$ for infinitely many $i$.

Remark. In the case where $Q$ is a regular local ring and $(\boldsymbol{f})$ is a $Q$-regular sequence, the ideals $\operatorname{Ann}_{\mathcal{R}} \mathcal{E}$ and $I_{n} \cap I_{n+1}$ both define the support set (or support variety, as it is referred to in [2]), so they have the same radical. However, they may not be the same ideal. Either one may be non-radical. It is not clear how their respective scheme structures differ; nor is it clear whether $I_{n} \cap I_{n+1}=I_{n^{\prime}} \cap I_{n^{\prime}+1}$ for $n \neq n^{\prime}$. 
Before proving the theorems, we make a few observations. We assume $Q$ is a domain.

It is easy to see that $X_{1}-a_{1}, \ldots, X_{c}-a_{c}$ is a regular sequence on $M[\boldsymbol{X}]$ (and $N[\boldsymbol{X}]$ ). We claim moreover that it is a regular sequence on $S$ provided $\overline{\boldsymbol{a}}=\left(\bar{a}_{1}, \ldots, \bar{a}_{c}\right)$ is nonzero in $k^{c}$. Indeed, assume $\overline{\boldsymbol{a}} \neq 0$. Note that

$$
\begin{aligned}
& S /\left(X_{1}-a_{1}, \ldots, X_{i}-a_{i}\right) \\
& \simeq Q\left[X_{i+1}, \ldots, X_{c}\right] /\left(a_{1} f_{1}+\cdots+a_{i} f_{i}+X_{i+1} f_{i+1}+\cdots+X_{c} f_{c}\right)
\end{aligned}
$$

for $i=1, \ldots, c-1$. Fix $i, 0 \leq i \leq c-1$, and suppose that for some $g, h \in Q\left[X_{i+1}, \ldots, X_{c}\right]$,

$$
g\left(X_{i+1}-a_{i+1}\right)=h\left(a_{1} f_{1}+\cdots+a_{i} f_{i}+X_{i+1} f_{i+1}+\cdots+X_{c} f_{c}\right)
$$

in $Q\left[X_{i+1}, \ldots, X_{c}\right]$. Since $X_{i+1}-a_{i+1}$ is prime in $Q\left[X_{i+1}, \ldots, X_{c}\right]$, either $X_{i+1}-a_{i+1}$ divides $h$ or $X_{i+1}-a_{i+1}$ divides $a_{1} f_{1}+\cdots+a_{i} f_{i}+X_{i+1} f_{i+1}+$ $\cdots+X_{c} f_{c}$. If it divided the latter, then $g_{1}\left(X_{i+1}-a_{i+1}\right)=a_{1} f_{1}+\cdots+a_{i} f_{i}+$ $X_{i+1} f_{i+1}+\cdots+X_{c} f_{c}$ for some $g_{1} \in Q\left[X_{i+1}, \ldots, X_{c}\right]$. But this equation clearly fails to hold for $0 \leq i \leq c-2$, and in the $i=c-1$ case, equating coefficients shows that $g_{1}=f_{c}$, which yields $-a_{c} f_{c}=a_{1} f_{1}+\cdots+a_{c-1} f_{c-1}$. However, since $f_{1}, \ldots, f_{c}$ is a minimal generating set for $\left(f_{1}, \ldots, f_{c}\right)$, this can only happen if $a_{i} \in \mathfrak{n}$ for all $i$, which contradicts $\boldsymbol{a} \neq 0$. Hence $X_{i+1}-a_{i+1}$ must divide $h$. By cancelling off the $X_{i+1}-a_{i+1}$ factors in $(*)$ we are left with

$$
g=h_{1}\left(a_{1} f_{1}+\cdots+a_{i} f_{i}+X_{i+1} f_{i+1}+\cdots+X_{c} f_{c}\right)
$$

for some $h_{1} \in Q\left[X_{i+1}, \ldots, X_{c}\right]$, which is what we needed to show in order to establish the claim.

We have the following basic isomorphisms of Ext and Tor modules (see, for example, p. 140 of [9]).

2.3. . Let $B:=A /(a)$ be rings with $a$ a nonzerodivisor on $A$. Suppose that $X$ and $Y$ are $A$-modules where $a$ is $X$-regular and $a Y=0$. Then for all $i$

$$
\operatorname{Ext}_{A}^{i}(X, Y) \simeq \operatorname{Ext}_{B}^{i}(X / a X, Y)
$$

and

$$
\operatorname{Tor}_{i}^{A}(X, Y) \simeq \operatorname{Tor}_{i}^{B}(X / a X, Y)
$$

As a last preparation we recall the change of rings long exact sequences of Ext (11.65 of [10]) and Tor (11.64 of [10]). 
2.4. . Let $B=A /(a)$ with $a$ a nonzerodivisor on $A$. Suppose that $X$ and $Y$ are $B$-modules. Then there is a long exact sequence of Ext

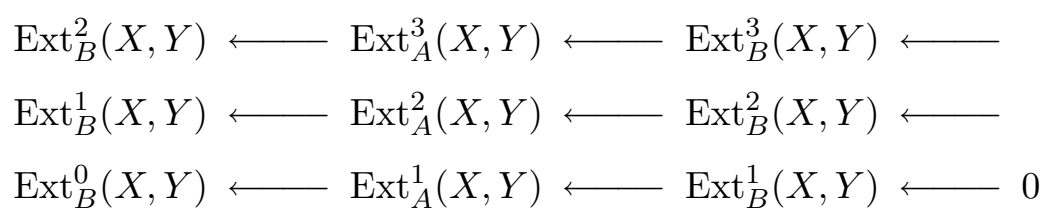

and a long exact sequence of Tor

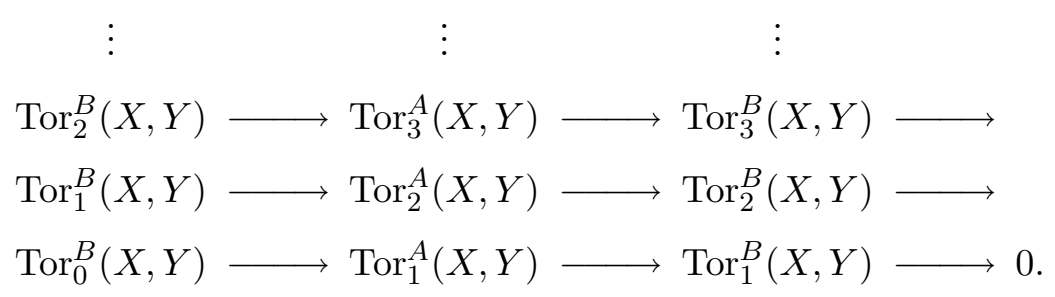

Proof of Theorem 2.1.

(1) If $\operatorname{Ext}_{Q}^{i}(M, N) \neq 0$ for infinitely many $i$, then 2.4 with $A=Q$ and $B=Q_{\boldsymbol{a}}$ shows that $\operatorname{Ext}_{Q_{\boldsymbol{a}}}^{i}(M, N) \neq 0$ for infinitely many $i$ and for all $Q_{\boldsymbol{a}}$, which means $\mathrm{V}(Q, \boldsymbol{f} ; M, N)=k^{c}$.

(2) Suppose that $\boldsymbol{\alpha}:=\left(\alpha_{1}, \ldots, \alpha_{c}\right) \in k^{c}$ is a nonzero element in $\mathrm{Z}\left(I_{n} \cap\right.$ $\left.I_{n+1}\right)$. Then the maximal ideal $p=\left(x_{1}-\alpha_{1}, \ldots, x_{c}-\alpha_{c}\right) \in \operatorname{Spec}(S / \mathfrak{n} S)$ is such that $p \supseteq I_{n} \cap I_{n+1}$. Hence $p \supseteq I_{n}$ or $p \supseteq I_{n+1}$. Without loss of generality, assume that $p \supseteq I_{n}$. Let $\boldsymbol{a}:=\left(a_{1}, \ldots, a_{c}\right) \in Q^{c}$ be any preimage of $\boldsymbol{\alpha}$. Then the maximal ideal $P:=\left(X_{1}-a_{1}, \ldots, X_{c}-a_{c}\right)+\mathfrak{n} S \in \operatorname{Spec}(S)$ is a preimage of $p$, and by Nakayama's Lemma, $\operatorname{Ext}_{S}^{n}(M[\boldsymbol{X}], N[\boldsymbol{X}])_{P} \neq 0$.

For $i=0, \ldots, c-1$ set $N_{i}:=N[\boldsymbol{X}] /\left(X_{1}-a_{1}, \ldots, X_{i}-a_{i}\right) N[\boldsymbol{X}]$ (with $\left.N_{0}:=N[\boldsymbol{X}]\right)$. We have short exact sequences

$$
0 \rightarrow\left(N_{i}\right)_{P} \stackrel{X_{i+1}-a_{i+1}}{\longrightarrow}\left(N_{i}\right)_{P} \rightarrow\left(N_{i+1}\right)_{P} \rightarrow 0,
$$

which give rise to exact sequences

$$
\operatorname{Ext}_{S}^{n}\left(M[\boldsymbol{X}], N_{i}\right)_{P} \stackrel{X_{i+1}-a_{i+1}}{\longrightarrow} \operatorname{Ext}_{S}^{n}\left(M[\boldsymbol{X}], N_{i}\right)_{P} \rightarrow \operatorname{Ext}_{S}^{n}\left(M[\boldsymbol{X}], N_{i+1}\right)_{P} .
$$

We first want to show that $\operatorname{Ext}_{S}^{n}(M[\boldsymbol{X}], N)_{P} \neq 0$. It suffices to prove that if $\operatorname{Ext}_{S}^{n}\left(M[\boldsymbol{X}], N_{i}\right)_{P} \neq 0$ then $\operatorname{Ext}_{S}^{n}\left(M[\boldsymbol{X}], N_{i+1}\right)_{P} \neq 0(0 \leq i \leq$ $c-1)$. (The fact that $\operatorname{Ext}_{S}^{n}(M[\boldsymbol{X}], N[\boldsymbol{X}])_{P} \neq 0$ begins the induction.) If $\operatorname{Ext}_{S}^{n}\left(M[\boldsymbol{X}], N_{i}\right)_{P} \neq 0$, then by Nakayama's lemma, $\operatorname{Ext}_{S}^{n}\left(M[\boldsymbol{X}], N_{i}\right)_{P} \otimes_{S_{P}}$ $\left(S /\left(X_{i+1}-a_{i+1}\right)\right)_{P} \neq 0$. By the exact sequence (2.1.1), this last module is a submodule of $\operatorname{Ext}_{S}^{n}\left(M[\boldsymbol{X}], N_{i+1}\right)_{P}$. Therefore $\operatorname{Ext}_{S}^{n}\left(M[\boldsymbol{X}], N_{i+1}\right)_{P}$ is nonzero. 
Now that we know $\operatorname{Ext}_{S}^{n}(M[\boldsymbol{X}], N)_{P} \neq 0$, we apply 2.3 inductively to arrive at $\operatorname{Ext}_{Q_{a}}^{n}(M, N) \neq 0$. If $\operatorname{Ext}_{Q_{a}}^{i}(M, N)=0$ for all $i \gg 0$, then from the change of rings long exact sequence 2.4 with $A=Q$ and $B=Q_{\boldsymbol{a}}$, $\operatorname{Ext}_{Q_{\boldsymbol{a}}}^{i}(M, N)=0$ for all $i \geq v-1$. But this contradicts the fact that $\operatorname{Ext}_{Q_{a}}^{n}(M, N) \neq 0$, as $n \geq v-1$. Hence there must exist infinitely many nonzero $\operatorname{Ext}_{Q_{\boldsymbol{a}}}^{i}(M, N)$. This establishes half of (2).

Now suppose that $\boldsymbol{\alpha}:=\left(\alpha_{1}, \ldots, \alpha_{c}\right) \neq 0$ is not in $\mathrm{Z}\left(I_{n} \cap I_{n+1}\right)$. Then the maximal ideal $p:=\left(x_{1}-\alpha_{1}, \ldots, x_{c}-\alpha_{c}\right) \in \operatorname{Spec}(S / \mathfrak{n} S)$ contains neither $I_{n}$ nor $I_{n+1}$. Let $\boldsymbol{a}:=\left(a_{1}, \ldots, a_{c}\right) \in Q^{c}$ be any preimage of $\boldsymbol{\alpha}$. Then the maximal ideal $P:=\left(X_{1}-a_{1}, \ldots, X_{c}-a_{c}\right)+\mathfrak{n} S \in \operatorname{Spec}(S)$ is a preimage of $p$, and by Nakayama's Lemma, $\operatorname{Ext}_{S}^{n}(M[\boldsymbol{X}], N[\boldsymbol{X}])_{P}=\operatorname{Ext}_{S}^{n+1}(M[\boldsymbol{X}], N[\boldsymbol{X}])_{P}=$ 0 . Note that since $Q \rightarrow Q[\boldsymbol{X}]$ is a flat extension, our assumption that $\operatorname{Ext}_{Q}^{i}(M, N)=0$ for all $i \geq v$ implies $\operatorname{Ext}_{Q[\boldsymbol{X}]}^{i}(M[\boldsymbol{X}], N[\boldsymbol{X}])=0$ for all $i \geq$ $v$. Now by 2.4 with $A=Q[\boldsymbol{X}]$ and $B=S$ we see that $\operatorname{Ext}_{S}^{i}(M[\boldsymbol{X}], N[\boldsymbol{X}])_{P}$ $=0$ for all $i \geq v-1$. From the short exact sequences (2.1.1), we inductively arrive at $\operatorname{Ext}_{S}^{i}(M[\boldsymbol{X}], N)_{P}=0$ for all $i \gg 0$. That is, $\operatorname{Ext}_{Q_{\boldsymbol{a}}}^{i}(M, N)=0$ for all $i \gg 0$ (by 2.3). Since $\boldsymbol{a}$ is an arbitrary preimage of $\boldsymbol{\alpha}$, we conclude that $\boldsymbol{\alpha}$ is not in $\mathrm{V}(Q, \boldsymbol{f} ; M, N)$.

For the final statement of Thereom 2.1, suppose $\operatorname{Ext}_{Q_{b}}^{i}(M, N) \neq 0$ for infinitely many $i$. Then $\boldsymbol{\alpha}$ is in $\mathrm{V}(Q, \boldsymbol{f} ; M, N)=\mathrm{Z}\left(I_{n} \cap I_{n+1}\right)$, and the first part of the proof shows that there exist infinitely many nonzero $\operatorname{Ext}_{Q_{a}}^{i}(M, N)$.

The Proof of Theorem 2.2 is exactly the same as the Proof of 2.1 .

\section{Properties of the support set.}

In this section we give various properties of support sets, beginning with:

\section{Independence of generating set.}

We first show that the support sets $\mathrm{V}(Q, \boldsymbol{f} ; M, N)$ and $\mathrm{U}(Q, \boldsymbol{f} ; M, N)$ are defined independently of the generating set $f$.

Proposition 3.1. Let $f^{\prime}:=f_{1}^{\prime}, \ldots, f_{c}^{\prime}$ be another minimal generating set for $(\boldsymbol{f})$. Then there exists an isomorphism $\tau: k^{c} \rightarrow k^{c}$ such that $\tau(\mathrm{V}(Q, \boldsymbol{f}$; $M, N))=\mathrm{V}\left(Q, \boldsymbol{f}^{\prime} ; M, N\right)$ and $\tau(\mathrm{U}(Q, \boldsymbol{f} ; M, N))=\mathrm{U}\left(Q, \boldsymbol{f}^{\prime} ; M, N\right)$.

Proof. Set

$$
S:=Q\left[X_{1}, \ldots, X_{c}\right] /\left(X_{1} f_{1}+\cdots+X_{c} f_{c}\right),
$$

and

$$
S^{\prime}:=Q\left[Y_{1}, \ldots, Y_{c}\right] /\left(Y_{1} f_{1}^{\prime}+\cdots+Y_{c} f_{c}^{\prime}\right)
$$


For $1 \leq j \leq c$ write

$$
f_{j}^{\prime}=\sum_{i=1}^{c} a_{j i} f_{i} \quad \text { with } \quad a_{j i} \in Q .
$$

Then we have a $Q$-linear ring homomorphism $\sigma: S \rightarrow S^{\prime}$ defined by

$$
X_{i} \mapsto \sum_{j=1}^{c} a_{j i} Y_{j} .
$$

Since both $\boldsymbol{f}$ and $\boldsymbol{f}^{\prime}$ are minimal generating sets of their ideal, the matrix $\left(a_{i j}\right)$ is invertible (over $Q$ ). Hence so is its transpose $\left(a_{j i}\right)$. This means $\sigma$ is a $Q$-algebra isomorphism. Let $\sigma^{\prime}$ denote its inverse.

We also have that the map $\rho: S / \mathfrak{n} S \rightarrow S^{\prime} / \mathfrak{n} S^{\prime}$ defined by

$$
x_{i} \mapsto \sum_{j=1}^{c} \bar{a}_{j i} y_{j}
$$

is an ismorphism of $k$-algebras. Let $\rho^{\prime}$ denote its inverse.

Set

$$
I_{i}:=\operatorname{Ann}_{S / \mathfrak{n} S}\left(\operatorname{Ext}_{S}^{i}(M[\boldsymbol{X}], N[\boldsymbol{X}]) \otimes_{S} S / \mathfrak{n} S\right)
$$

and

$$
I_{i}^{\prime}:=\operatorname{Ann}_{S^{\prime} / \mathfrak{n} S^{\prime}}\left(\operatorname{Ext}_{S^{\prime}}^{i}(M[\boldsymbol{Y}], N[\boldsymbol{Y}]) \otimes_{S^{\prime}} S^{\prime} / \mathfrak{n} S^{\prime}\right) .
$$

We claim that $\rho^{\prime}\left(I_{i}^{\prime}\right)=I_{i}$. To see this, note that we have the isomorphism $\mu: M[\boldsymbol{X}] \rightarrow M[\boldsymbol{Y}]$ defined by $X_{i} \mapsto \sum_{j=1}^{c} a_{j i} Y_{j}$ (which is $\sigma$-equivariant). Taking the inverse of this, with $M$ replaced by $N$, we also have an isomorphism $\nu: N[\boldsymbol{Y}] \rightarrow N[\boldsymbol{X}]$ (which is $\sigma$-equivariant). The upshot is that these induce isomorphisms

$$
\epsilon_{i}: \operatorname{Ext}_{S}^{i}(M[\boldsymbol{X}], N[\boldsymbol{X}]) \otimes_{S} S / \mathfrak{n} S \rightarrow \operatorname{Ext}_{S^{\prime}}^{i}(M[\boldsymbol{Y}], N[\boldsymbol{Y}]) \otimes_{S^{\prime}} S^{\prime} / \mathfrak{n} S^{\prime}
$$

and

$$
\epsilon_{i}^{\prime}: \operatorname{Ext}_{S^{\prime}}^{i}(M[\boldsymbol{Y}], N[\boldsymbol{Y}]) \otimes_{S^{\prime}} S^{\prime} / \mathfrak{n} S^{\prime} \rightarrow \operatorname{Ext}_{S}^{i}(M[\boldsymbol{X}], N[\boldsymbol{X}]) \otimes_{S} S / \mathfrak{n} S,
$$

which are $\rho$-equivariant.

Let $\psi$ be in $I_{i}^{\prime}$. Let $e$ be an arbitrary element of $\operatorname{Ext}_{S}^{i}(M[\boldsymbol{X}], N[\boldsymbol{X}]) \otimes_{S}$ $S / \mathfrak{n} S$. Then there exists $e^{\prime} \in \operatorname{Ext}_{S^{\prime}}^{i}(M[\boldsymbol{Y}], N[\boldsymbol{Y}]) \otimes_{S^{\prime}} S^{\prime} / \mathfrak{n} S^{\prime}$ such that $\epsilon^{\prime}\left(e^{\prime}\right)=e$. We have $\rho^{\prime}(\psi) \cdot e=\rho^{\prime}(\psi) \cdot \epsilon^{\prime}\left(e^{\prime}\right)=\psi \cdot \epsilon^{\prime}\left(e^{\prime}\right)=\epsilon^{\prime}\left(\psi \cdot e^{\prime}\right)=\epsilon^{\prime}(0)=0$. Therefore $\rho^{\prime}(\psi) \in I_{i}$, and we have shown $\rho^{\prime}\left(I_{i}^{\prime}\right) \subseteq I_{i}$. By symmetry, $\rho\left(I_{i}\right) \subseteq$ $I_{i}^{\prime}$, and applying $\rho^{\prime}$ gives the reverse inclusion. This establishes the claim.

Let the matrix $\left(\bar{b}_{j i}\right)\left(b_{j i} \in Q\right)$ be the inverse of the matrix $\left(\bar{a}_{j i}\right)$, so that for $1 \leq i \leq c$ we have $\rho^{\prime}\left(y_{i}\right)=\sum_{j=1}^{c} \bar{b}_{j i} x_{j}$. Representing elements of $k^{c}$ as column vectors, we define $\tau: k^{c} \rightarrow k^{c}$ by

$$
\tau\left(\left[\begin{array}{c}
\alpha_{1} \\
\vdots \\
\alpha_{c}
\end{array}\right]\right)=\left(\bar{b}_{j i}\right)\left[\begin{array}{c}
\alpha_{1} \\
\vdots \\
\alpha_{c}
\end{array}\right] .
$$


Now we are ready to show that $\tau(\mathrm{V}(Q, \boldsymbol{f} ; M, N))=\mathrm{V}\left(Q, \boldsymbol{f}^{\prime} ; M, N\right)$.

Choose $\left[\begin{array}{c}\alpha_{1} \\ \vdots \\ \alpha_{c}\end{array}\right] \in \mathrm{Z}\left(I_{i} \cap I_{i+1}\right)$ and $\psi\left(y_{1}, \ldots, y_{c}\right) \in I_{i}^{\prime} \cap I_{i+1}^{\prime}$. Let $\phi\left(x_{1}, \ldots, x_{c}\right)$ $\in I_{i} \cap I_{i+1}$ be such that $\rho\left(\phi\left(x_{1}, \ldots, x_{c}\right)\right)=\psi\left(y_{1}, \ldots, y_{c}\right)$. This equation can be represented by

$$
\phi\left(\left(\bar{a}_{j i}\right)\left[\begin{array}{c}
x_{1} \\
\vdots \\
x_{c}
\end{array}\right]\right)=\psi\left(\left[\begin{array}{c}
y_{1} \\
\vdots \\
y_{c}
\end{array}\right]\right) .
$$

We have

$$
\psi\left(\left(\bar{b}_{j i}\right)\left[\begin{array}{c}
\alpha_{1} \\
\vdots \\
\alpha_{c}
\end{array}\right]\right)=\phi\left(\left(\bar{a}_{j i}\right)\left(\bar{b}_{j i}\right)\left[\begin{array}{c}
\alpha_{1} \\
\vdots \\
\alpha_{c}
\end{array}\right]\right)=\phi\left(\left[\begin{array}{c}
\alpha_{1} \\
\vdots \\
\alpha_{c}
\end{array}\right]\right)=0 .
$$

Hence $\tau\left(\left[\begin{array}{c}\alpha_{1} \\ \vdots \\ \alpha_{c}\end{array}\right]\right) \in \mathrm{Z}\left(I_{i}^{\prime} \cap I_{i+1}^{\prime}\right)$. The conclusion follows by symmetry.

The proof that $\tau(\mathrm{U}(Q, \boldsymbol{f} ; M, N))=\mathrm{U}\left(Q, \boldsymbol{f}^{\prime} ; M, N\right)$ is completely analogous.

\section{Projective Dimension and Nontriviality of Support Sets.}

We now discuss a particular case of interest, namely, when $N=k$. In this case the support sets $\mathrm{V}(Q, \boldsymbol{f} ; M):=\mathrm{V}(Q, \boldsymbol{f} ; M, k)$ and $\mathrm{U}(Q, \boldsymbol{f} ; M):=$ $\mathrm{U}(Q, \boldsymbol{f} ; M, k)$ describe those elements $h \in(\boldsymbol{f})-\mathfrak{n}(\boldsymbol{f})$ such that $M$ has infinite projective dimension over $Q /(h)$. The next proposition shows that often it is enough to study this special case. The proof of (1) is identical to the proof of part (8) of Theorem 5.6 of [2], but we include it here since it is short.

Proposition 3.2. Assume that $Q$ is a regular local ring. Then we have

$$
\mathrm{V}(Q, \boldsymbol{f} ; M, N)=\mathrm{V}(Q, \boldsymbol{f} ; M) \cap \mathrm{V}(Q, \boldsymbol{f} ; N)
$$

and

$$
\mathrm{U}(Q, \boldsymbol{f} ; M, N)=\mathrm{U}(Q, \boldsymbol{f} ; M) \cap \mathrm{U}(Q, \boldsymbol{f} ; N) .
$$

Proof. Suppose that $0 \neq \boldsymbol{\alpha} \in \mathrm{V}(Q, \boldsymbol{f} ; M, N)$. Then $\operatorname{Ext}_{Q \boldsymbol{a}}^{i}(M, N) \neq 0$ for infinitely many $i$ where $\boldsymbol{a} \in Q^{c}$ is a preimage of $\boldsymbol{\alpha}$. Hence it must be the case that $M$ has infinite projective dimension over $Q_{\boldsymbol{a}}$ and $N$ has infinite injective dimension over $Q_{\boldsymbol{a}}$. Since $Q_{\boldsymbol{a}}$ is Gorenstein, $N$ has infinite injective dimension over $Q_{a}$ if and only if it has infinite projective dimension over $Q_{\boldsymbol{a}}$. Thus $\boldsymbol{\alpha} \in \mathrm{V}(Q, \boldsymbol{f} ; M) \cap \mathrm{V}(Q, \boldsymbol{f} ; N)$.

Now suppose $0 \neq \boldsymbol{\alpha} \notin \mathrm{V}(Q, \boldsymbol{f} ; M, N)$. This means that $\operatorname{Ext}_{Q \boldsymbol{a}}^{i}(M, N)=0$ for all $i \gg 0$. By 5.12 of [2], either $M$ or $N$ has finite projective dimension over $Q_{\boldsymbol{a}}$, where $\boldsymbol{a}$ a preimage of $\boldsymbol{\alpha}$.

The proof for homological support sets is similar, using a result of Huneke and Wiegand this time $((1.9)$ of $[6])$, which states that if $\operatorname{Tor}_{i}^{Q a}(M, N)=0$ 
for all $i \gg 0$, then either $M$ or $N$ has finite projective dimension over the hypersurface $Q_{a}$.

It is quite common that the support set is all of $k^{c}$ :

Example 3.3. Suppose $(\boldsymbol{f}) \subseteq \mathfrak{n}^{2}$. Then the residue field $k$ has full support set $\mathrm{V}(Q, \boldsymbol{f} ; k)=k^{c}$.

This follows from the well-known fact of Auslander-Buchsbaum-Serre, that the projective dimension of the residue field of a local ring $S$ is finite if and only if $S$ is regular. If $(\boldsymbol{f}) \subseteq \mathfrak{n}^{2}$, then the hypersurfaces $Q_{\boldsymbol{a}}$ are never regular.

When $\boldsymbol{f}$ is a $Q$-regular sequence we have $\mathrm{V}(Q, \boldsymbol{f} ; R)=\{0\}$. However, for non-regular sequences $(\boldsymbol{f})$, it may be the case that the cohomological support set of even this pair $(R, k)$ is the whole space $k^{c}$, as the next example shows.

Example 3.4. Let $Q=k[[X, Y, Z]]$ and $(\boldsymbol{f})=(X Y, Y Z)$. Then $\mathrm{V}(Q, \boldsymbol{f} ; R)$ $=k^{2}$.

Proof. We just need to show that $R$ has infinite projective dimension over each of the hypersurfaces $Q_{\boldsymbol{a}}:=Q /\left(a_{1} X Y+a_{2} Y Z\right)$ with $\boldsymbol{a}:=\left(a_{1}, a_{2}\right) \in Q^{2}$ a preimage of a nonzero $\boldsymbol{\alpha} \in k^{2}$.

If $\boldsymbol{\alpha} \neq 0$, then for any preimage $\boldsymbol{a}:=\left(a_{1}, a_{2}\right)$, either $a_{1}$ or $a_{2}$ is a unit in $Q$. Without loss of generality assume that $a_{1}$ is a unit. Then $R \simeq Q_{\boldsymbol{a}} /(y z)$ (where $y$ denotes the image of $Y$ in $Q_{a}$, etc.), and one can easily check that a $Q_{a}$-free resolution of $R$ is given by

$$
\ldots \stackrel{\left[a_{1} x+a_{2} z\right]}{\longrightarrow} Q_{\boldsymbol{a}} \stackrel{[y]}{\longrightarrow} Q_{\boldsymbol{a}} \stackrel{\left[a_{1} x+a_{2} z\right]}{\longrightarrow} Q_{\boldsymbol{a}} \stackrel{[y z]}{\longrightarrow} Q_{\boldsymbol{a}} \longrightarrow R \longrightarrow 0,
$$

which is infinite (and periodic after the first step).

3.5. . We remark here that Example 4.4 of the next section shows that the codimension three Gorenstein ring

$$
R:=k\left[\left[X_{1}, \ldots, X_{5}\right]\right] /\left(X_{1} X_{3}, X_{1} X_{4}, X_{2} X_{4}, X_{2} X_{5}, X_{3} X_{5}\right)
$$

also has full support set $\mathrm{V}(Q, \boldsymbol{f} ; R)=k^{5}$.

The next proposition gives a general source of modules without full support set.

Proposition 3.6. Let $M^{\prime}$ be a module over the regular local ring $Q$, and let $I$ be an ideal of $Q$ contained in $\operatorname{Ann}_{Q} M^{\prime}$. Assume that $f \in \mathfrak{n}$ is regular on $M^{\prime}$. Let $f_{2}, \ldots, f_{c}$ be a minimal generating set for $I$. Then $f, f_{2}, \ldots, f_{c}$ is a minimal generating set for $(f)+I$, and for the $R:=Q /(f)+I$-module $M:=M^{\prime} / f M^{\prime}$ we have $\mathrm{V}\left(Q, f, f_{2}, \ldots, f_{c} ; M\right) \neq k^{c}$. 
Proof. Since $Q$ is a regular local ring we know that $M^{\prime}$ has a finite $Q$-free resolution $\mathbf{F}$, and since $f$ is both $Q$-regular and $M^{\prime}$-regular, $\mathbf{F} \otimes_{Q} Q /(f)$ is a finite free resolution of $M$ over $Q /(f)$. Thus the $R$-module $M$ has finite projective dimension over the hypersurface $Q /(f)$ with $f \in(f)+I-\mathfrak{n}((f)+$ $I)$, and so $\mathrm{V}\left(Q, f, f_{2}, \ldots, f_{c} ; M\right) \neq k^{c}$.

Remark. Proposition 3.6 does not describe the only way for modules to have a non-full support set. Example 4.5 of the next section illustrates that the ring $R:=k\left[\left[X_{1}, X_{2}, X_{3}, X_{4}\right]\right] /\left(X_{1} X_{4}, X_{2} X_{4}, X_{2}^{2}-X_{4}^{2}, X_{1}^{2}-X_{2}^{2}, X_{1} X_{2}-\right.$ $\left.X_{3} X_{4}\right)$ has finite projective dimension over the hypersurface $R:=k\left[\left[X_{1}, X_{2}\right.\right.$, $\left.\left.X_{3}, X_{4}\right]\right] /\left(X_{1} X_{2}-X_{3} X_{4}\right)$. However, $R$ does not lift to $k\left[\left[X_{1}, X_{2}, X_{3}, X_{4}\right]\right]$, meaning that there is no $M^{\prime}$ such that $X_{1} X_{2}-X_{3} X_{4}$ is $M^{\prime}$-regular and $M^{\prime} /\left(X_{1} X_{2}-X_{3} X_{4}\right) M^{\prime} \simeq R$ (cf. [8]).

\section{Respecting Theorem AB.}

Example 3.4 shows that implications (1), (2), (3) $\Longrightarrow(4)$ of Theorem AB can fail utterly using our notion of support set for quotient rings $R=Q /(\boldsymbol{f})$ not defined modulo a regular sequence, and Example 4.4 shows that these implications can fail even when $R$ is Gorenstein. However, it is an open question whether the reverse implications hold:

(*) Suppose $Q$ is a regular local ring. Do the implications (4) $\Longrightarrow(1),(2)$, (3) of Theorem $\mathrm{AB}$ hold for support sets defined over quotients $R:=Q /(\boldsymbol{f})$ in which $\boldsymbol{f}$ is not a regular sequence?

None of the apparatus used in the proof of Theorem AB is available to us when $\boldsymbol{f}$ is not a regular sequence. Hence any proof of $(*)$ would require a completely different approach.

It seems that the statement $\mathrm{V}(Q, \boldsymbol{f} ; M) \cap \mathrm{V}(Q, \boldsymbol{f} ; N)=\{0\}$ is a much more severe condition when $\boldsymbol{f}$ is not a regular sequence than when $\boldsymbol{f}$ is a regular sequence. In fact, we have no counterexample to the following stronger version of $(*)$.

Question 3.7. Suppose $Q$ is a regular local ring and that $M$ and $N$ are $R$-modules with $\mathrm{V}(Q, \boldsymbol{f} ; M, N)=\{0\}$. Must $R$ then be a complete intersection?

\section{Macaulay 2 code and examples.}

In this section we give code for Macaulay 2 [4], written by Amelia Taylor ${ }^{2}$ and the current author, that effectively employs the method of Theorem 2.2 to compute homological support sets. Simply replacing the Tors by Exts yields code for computing cohomological support sets, using Theorem 2.1.

\footnotetext{
${ }^{2}$ This research was conducted by the author for the Clay Mathematics Institute.
} 
The user will input a pair of modules defined over a quotient of a polynomial ring by an ideal minimally generated by $c$ elements. The output is an ideal of the polynomial ring $k\left[x_{1}, \ldots, x_{c}\right]$ that defines the support set. This ideal may or may not be radical (cf. the remark following Theorem 2.2).

We also give several examples illustrating its use.

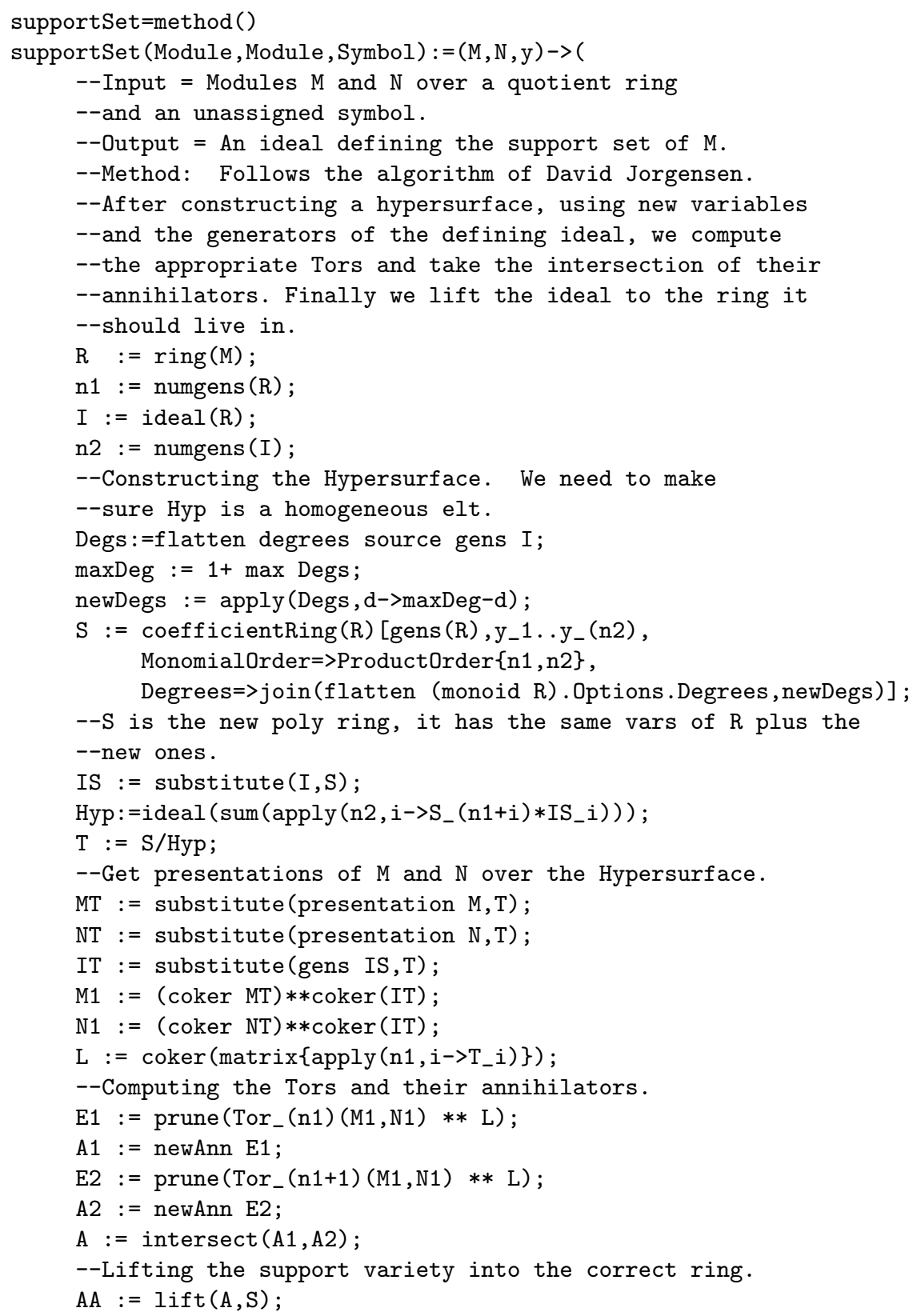




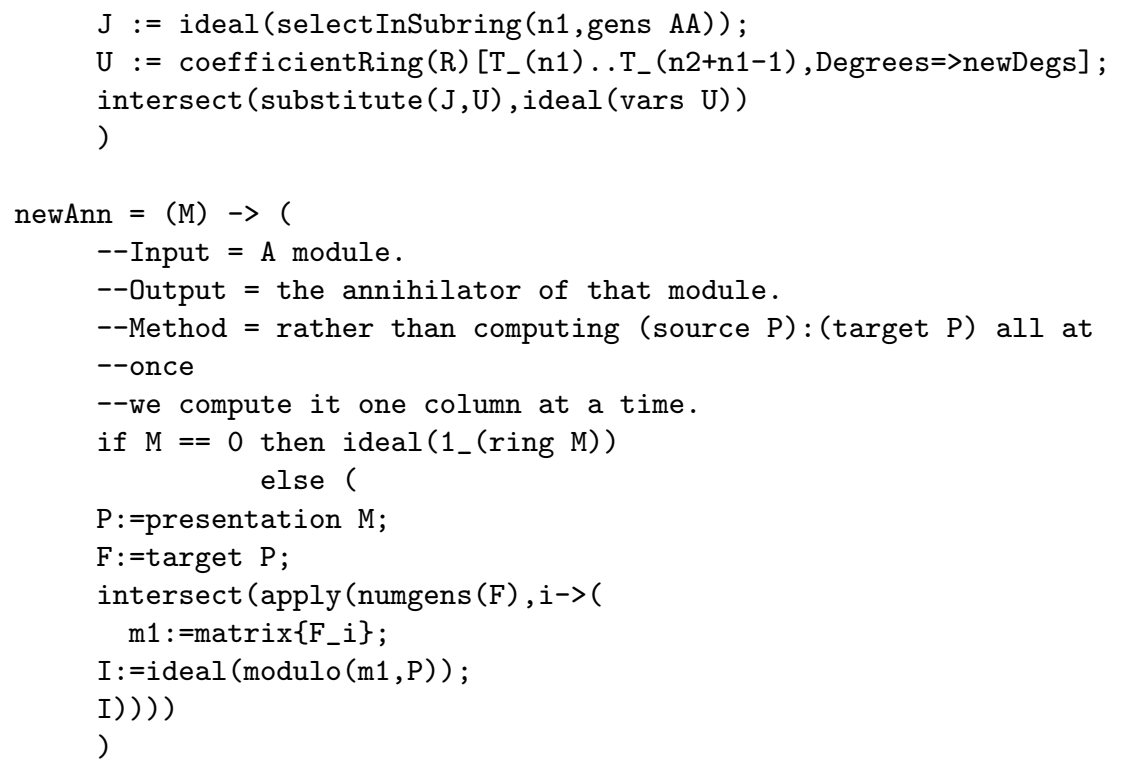

Example 4.1. First we give an example illustrating the fact that if $R$ is a complete intersection, then $\mathrm{V}(Q, \boldsymbol{f} ; R)=\{0\}$.

Macaulay 2, version 0.8 .99

--Copyright 1993-2001, all rights reserved, D. R. Grayson and M. E. --Stillman

--Factory 1.2c from Singular, copyright 1993-1997, G.-M. Greuel, R.

--Stobbe

--Factorization and characteristic sets 0.3.1, copyright 1996, M.

--Messollen

--GC 6.0 alpha 2, copyright, H-J. Boehm, A. Demers

--GNU C Library (glibc-2.1.3), copyright, Free Software Foundation

--GNU MP Library (gmp-3.1.1), copyright, Free Software Foundation

i1 : load"supportSet.m2"

--loaded supportSet.m2

i2 : $R=Q Q[a, b, c] / i d e a l\left(a^{\wedge} 2-b * c, b^{\wedge} 2-a * c, c^{\wedge} 2+a * b\right)$

o1 : R

01 : QuotientRing

i3 : $k=$ coker vars $R$

o3 = cokernel $\mid \mathrm{a}$ b c |

o3 : R-module, quotient of $R$ 


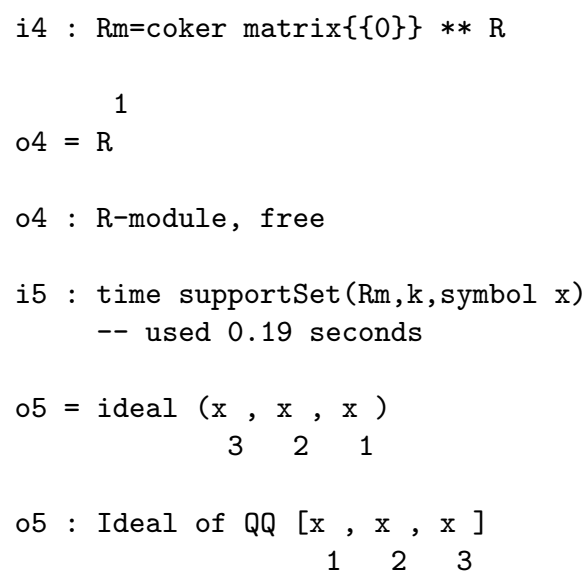

Example 4.2. We illustrate Example 3.3 (that $\mathrm{V}(Q, \boldsymbol{f} ; k)=k^{c}$ when $(\boldsymbol{f}) \subseteq$ $\left.\mathfrak{n}^{2}\right)$ for the current ring:

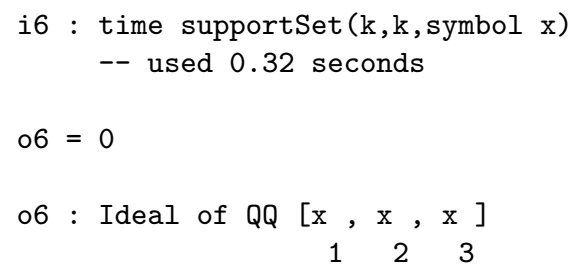

Example 4.3. Some modules have interesting support sets. Here is a module whose support set is defined by the ideal $\left(x^{2}-y z, y^{2}-x z, z^{2}-x y\right)$.

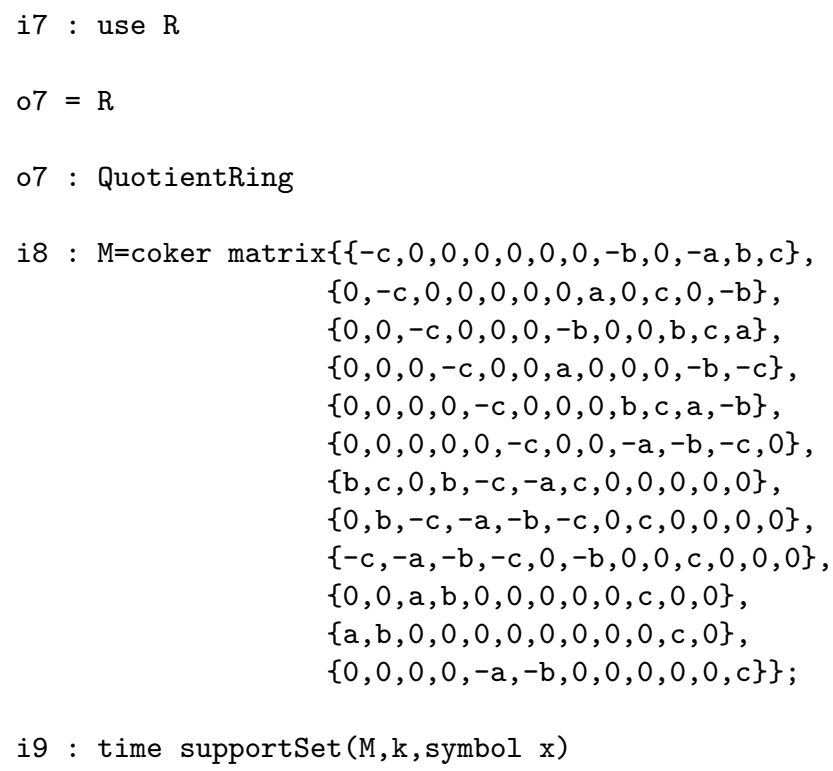


-- used 3.06 seconds

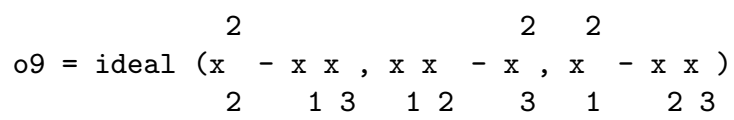

$$
\begin{aligned}
& \text { o9 : Ideal of } \mathrm{QQ}[\mathrm{x}, \mathrm{x}, \mathrm{x}] \\
& 123
\end{aligned}
$$

Example 4.4. We give a codimension three Gorenstein $\operatorname{ring} R$ with $\mathrm{V}(Q, \boldsymbol{f}$; $R)=k^{c}$.

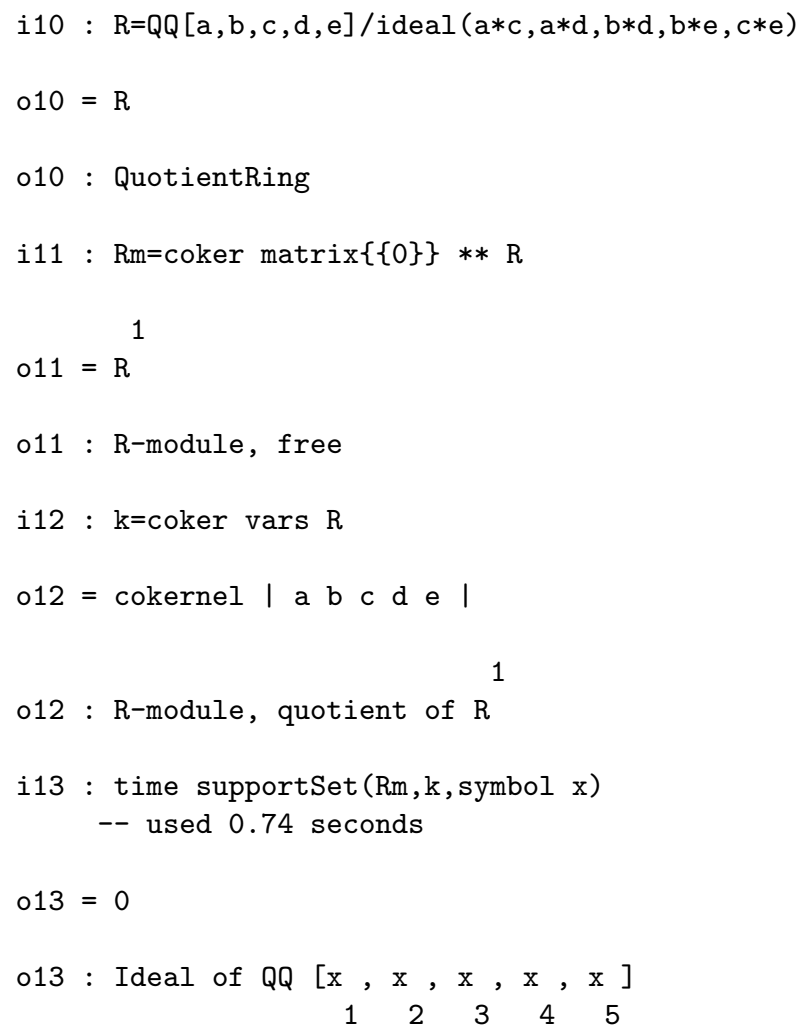

Example 4.5. In this example we indicate that the non-complete intersection ring $R:=k[[a, b, c, d]] /\left(a d, b d, b^{2}-d^{2}, a^{2}-b^{2}, a b-c d\right)$ has a nontrivial support set defined by $\left(x_{3}^{2}-x_{5}^{2}\right)$.

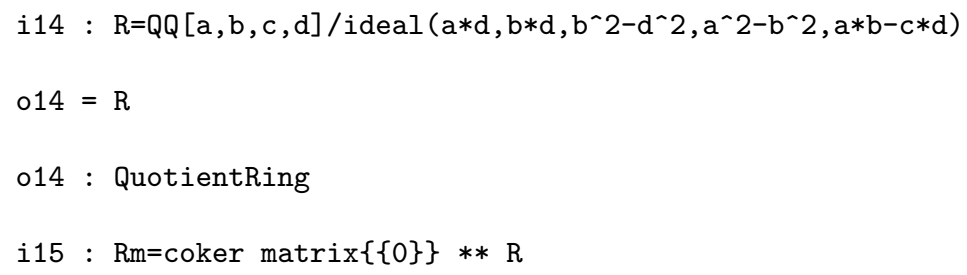




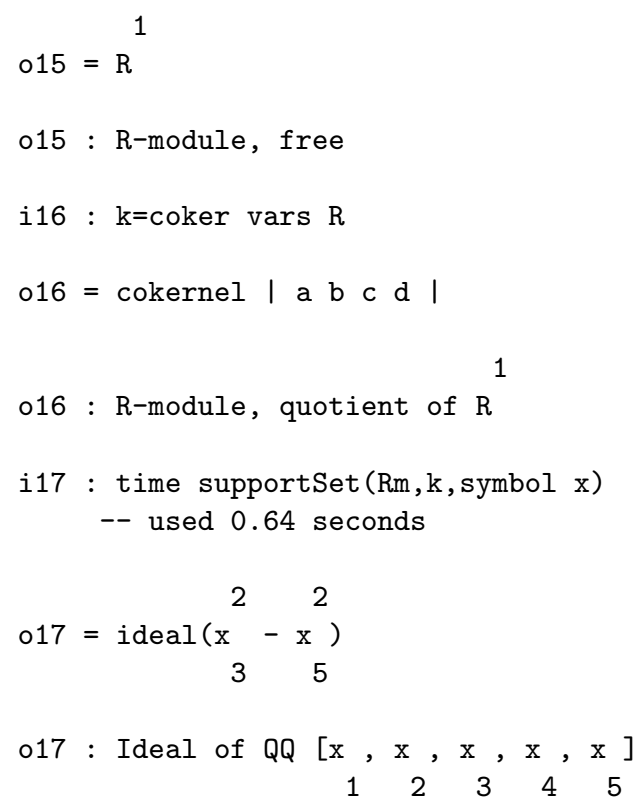

Example 4.6. Finally, we give an example of a one-dimensional support set over a non-complete intersection (cf. Question 3.6).

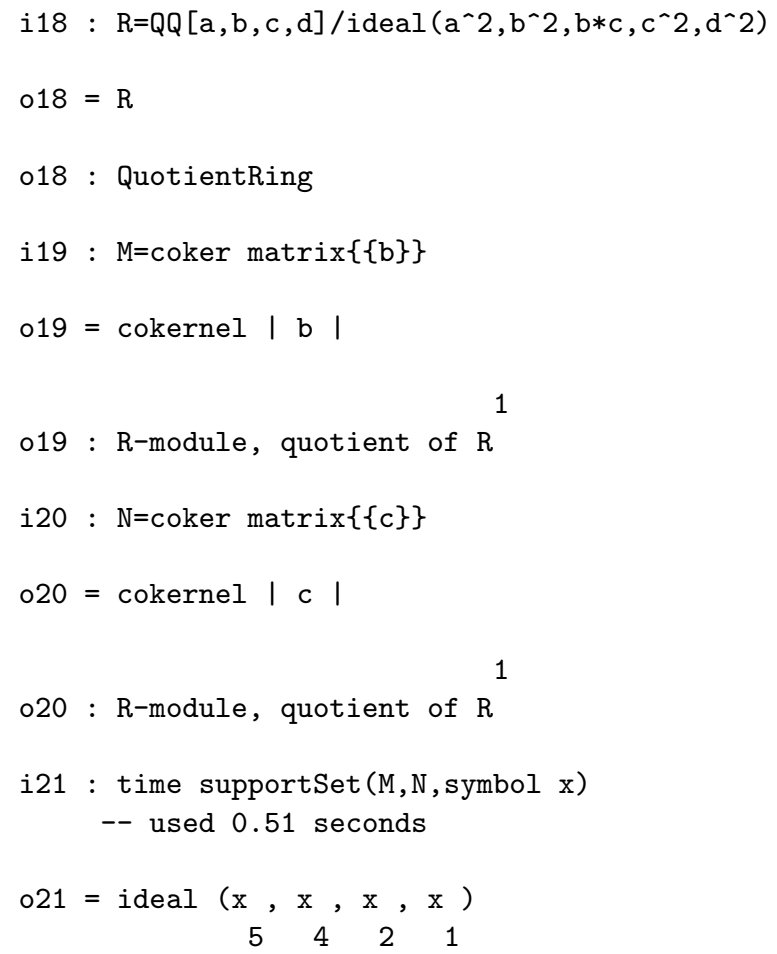




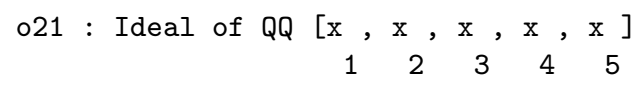

Acknowledgements. This research was conducted while I was visiting the University of Kansas. I thank the Department of Mathematics for their support and generosity.

I would like to thank Amelia Taylor for all her help in putting together and testing the Macaulay 2 script given here. I would also like to thank Craig Huneke for many interesting conversations regarding support varieties.

\section{References}

[1] L.L. Avramov, Modules of finite virtual projective dimension, Invent. Math., 96 (1989), 71-101, MR 90g:13027, Zbl 0677.13004.

[2] L.L. Avramov and R.-O. Buchweitz, Support varieties and cohomology over complete intersections, Invent. Math., 142(2) (2000), 285-318, MR 2001j:13017.

[3] N. Bourbaki, Algèbre Commutative. IX: Anneaux Locaux Réguliers Complets, Masson, Paris, 1983, Zbl 0579.13001.

[4] D. Grayson and M. Stillman, Macaulay 2, a software system for research in algebraic geometry, 1993.

[5] T.H. Gulliksen, A change of rings theorem, with applications to Poincaré series and intersection multiplicity, Math. Scand., 34 (1974), 167-183, MR 51 \#487, Zbl 0292.13009.

[6] C. Huneke and R. Wiegand, Tensor products of modules, rigidity and local cohomology, Math. Scand., 81 (1997), 161-183, MR 2000d:13027, Zbl 0908.13010.

[7] D.A. Jorgensen, Tor and torsion on a complete intersection, J. Algebra, 195 (1997), 526-537, MR 98k:13014, Zbl 0898.13008.

[8] _ Existence of unliftable modules, Proc. Amer. Math. Soc., 127(6) (1999), 1575-1582, MR 99i:13028, Zbl 0912.13004.

[9] H. Matsumura, Commutative Ring Theory, Cambridge University Press, 1986, MR 88h:13001, Zbl 0603.13001.

[10] J.J. Rotman, An Introduction to Homological Algebra, Academic Press, Inc., 1979, MR 80k:18001, Zbl 0441.18018.

Received March 27, 2001 and revised September 13, 2001.

Department of Mathematics

UNIVERSITY OF KANSAS

LAWRENCE, KS 66045

Department of Mathematics

University of Texas at Arlington

Arlington, TX 76019

E-mail address: djorgens@math.uta.edu 